\title{
Discovery and Development of Novel Salicylate Synthase (Mbtl) Furanic Inhibitors as Antitubercular Agents
}

Laurent R. Chiarelli, a, ${ }^{\prime}$ Matteo Mori, ${ }^{b, \neq}$ Daniela Barlocco, ${ }^{b}$ Giangiacomo Beretta, ${ }^{c}$ Arianna Gelain, ${ }^{b}$ Elena Pini, ${ }^{b}$ Marianna Porcino, ${ }^{b}$ Giorgia Mori, ${ }^{a}$ Giovanni Stelitano, ${ }^{a}$ Luca Costantino, ${ }^{d}$ Margherita Lapillo, ${ }^{e}$ Davide Bonanni, Giulio Poli, ${ }^{,}$Tiziano Tuccinardi, ${ }^{,}{ }^{*}$ Stefania Villa ${ }^{b,{ }^{*}}$ and Fiorella Meneghettib.

a Dipartimento di Biologia e Biotecnologie "Lazzaro Spallanzani", Università degli Studi di Pavia, via Ferrata 9, 27100 Pavia; Italy.

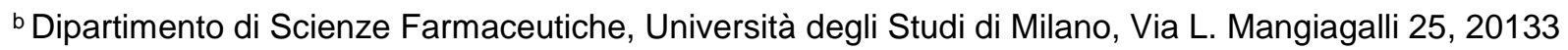
Milano; Italy.

c Dipartimento di Scienze e Politiche Ambientali, Università degli Studi di Milano, Via Celoria 2, 20133 Milano; Italy.

${ }^{d}$ Dipartimento Scienze della Vita, Università degli Studi di Modena e Reggio Emilia, via Campi 103, 41121 Modena; Italy.

e Dipartimento di Farmacia, Università di Pisa, via Bonanno 6, 56126 Pisa; Italy.

f Dipartimento di Biotecnologie, Chimica e Farmacia, Università di Siena, via A. Moro 2, 53100 Siena; Italy.

KEYWORDS: Tuberculosis, Iron, siderophores, antimycobacterial drugs, virtual screening, drug design 
ABSTRACT. We report on the virtual screening, synthesis, and biological evaluation of new furan derivatives targeting Mycobacterium tuberculosis salicylate synthase (Mbtl). A receptor-based virtual screening procedure was applied to screen the Enamine database, identifying two compounds, I and III, endowed with a good enzyme inhibitory activity. Considering the most active compound I as starting point for the development of novel Mbtl inhibitors, we obtained new derivatives based on the furan scaffold. Among the SAR performed on this class, compound 1a emerged as the most potent Mbtl inhibitor reported to date $\left(K_{\mathrm{i}}=5.3 \mu \mathrm{M}\right)$. Moreover, compound 1a showed a promising antimycobacterial activity $\left(\mathrm{MIC}^{99}=156 \mu \mathrm{M}\right)$, which is conceivably related to mycobactin biosynthesis inhibition.

\section{INTRODUCTION}

Tuberculosis (TB), caused by the pathogen Mycobacterium tuberculosis (Mtb), is one of the deadliest infectious diseases worldwide, with about 1.7 million deaths reported in 2016 [1]. New TB drugs are needed because of the complexity and toxicity of the current TB therapeutic regimens and to provide alternatives to contrast the emergence of multidrugresistant (MDR) and extensively drug-resistant (XDR) Mtb strains [2,3]. The investigation of novel pharmacological targets, such as InhA, MmpL3, DprE1 and QcrB, plays a fundamental role in the development of new potential antitubercular agents [4]. Since the most promising targets are membrane proteins, the identified hit compounds may also interfere with the integrity of the host membranes; therefore, the selection of different targets to be tackled is still urgent [5-8]. In addition, there are a number of novel anti-TB compounds in clinical trial, and two new drugs have been recently approved: delamanid and bedaquiline. Despite these advances, many more TB drug candidates are necessary to sustain an effective and productive drug pipeline [9]. In particular, more information on the effectiveness, safety and tolerability of bedaquiline is urgently required, because its severe side effects (mainly QT prolongation) could be potentially life-threatening [9]. Therefore, the research of new antiTB drugs that can shorten and simplify current pharmacological therapy is pivotal [10].

One of the strategies adopted to fight bacterial pathogens is to target the biosynthesis of mycobactins, high-affinity iron-chelating molecules called siderophores [6]. Iron is an essential redox cofactor, involved in several cellular processes, allowing the survival and growth of the mycobacterium in the host. Nevertheless, Mtb is capable of sustaining an infection even in an iron-deficient environment by overcoming it by means of several iron acquisition pathways, among which the synthesis of siderophores plays a key role. Water- 
soluble carboxymycobactins and lipophilic mycobactins, compounds that vary according to the nature of their lipophilic moiety, are able to solubilize iron and compete with the ironbinding proteins (e.g. transferrin, lactoferrin, ferritin) of the host. While mycobactins remain associated within the cell wall, carboxymycobactins are released outside the outer membrane, where they scavenge iron, making it available for transport into the Mtb cytoplasm [11]. As the bacterial strains unable to produce siderophores lose their ability to grow within macrophages and sustain the infection [12], there is a considerable interest in deciphering the mechanism of siderophore assembly, with the goal of targeting selected steps for antitubercular drug development. Among the enzymes involved in siderophore biosynthesis [6], the $\mathrm{Mg}^{2+}$-dependent salicylate synthase (Mbtl) has been validated as a target for fighting TB $[13,14]$. Since Mbtl catalyzes the first step of the process by converting chorismate to salicylate via an isochorismate intermediate (Scheme 1), its inhibition leads to a reduced mycobacterial pathogenicity without toxicity issues, due to the absence of this target in the host [15]. The first structural and biochemical characterization of Mbtl was reported by Harrison et al. [16] and structural data highlights that the $\mathrm{Mg}^{2+}$ is coordinated by E297 and E434, as well as the $C_{1}$ carboxylate of chorismate [13]. Residue K205 assists the attack of water at the chorismate $\mathrm{C}_{2}$ carbon for the isomerization reaction and $\mathrm{E} 252$ provides acid catalysis for the elimination of the hydroxyl group from $\mathrm{C}_{4}$ carbon. The positioning of the pyruvate side chain of the substrate is allowed by the interactions with Y385, R405 and G419 [13].

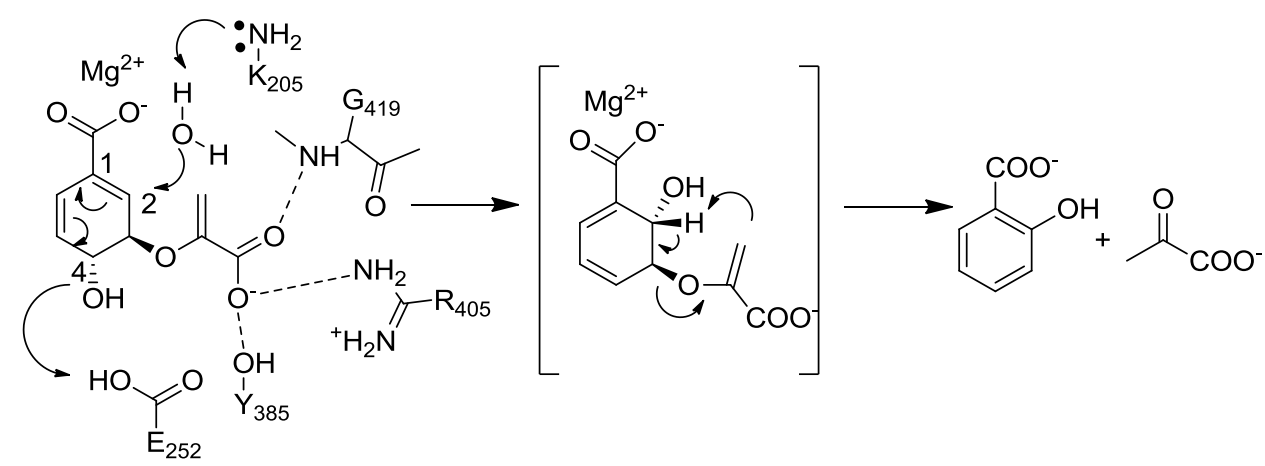

Scheme 1. Reaction catalyzed by Mbtl, the salicylate synthase of Mycobacterium tuberculosis.

With the aim of identifying new Mbtl inhibitors, a receptor-based virtual screening (VS) study was carried out and a preliminary optimization of one of the two identified hits was performed. This study has led to novel chemical entities that could represent an alternative 
and/or complementary therapy for the treatment of multidrug-resistant strains, opening new avenues to improved clinical outcomes.

\section{RESULTS AND DISCUSSION}

Virtual Screening. A VS study, focused on the ligand-Mbtl key interactions, was developed. A pharmacophore model was created based on the analysis of the crystal structure of Mbtl co-crystallized with 3-(1-carboxyprop-1-enyloxy)-2-hydroxybenzoic acid (Methyl-AMT, PDB code 3VEH) [17]. Figure 1A depicts the interactions of Methyl-AMT inside the Mbtl binding site. The carboxylic group of the 2-hydroxy benzoic acid forms an $\mathrm{H}$ bond with the side-chain of Y385; the analysis of different Mbtl crystal structures suggests that the position of this residue is highly conserved, and it is important for the interaction of the carboxylic function of the reported Mbtl inhibitors. The aromatic ring of the 2-hydroxy benzoic acid shows lipophilic interactions with 1207, A362, L404 and a cation-m interaction with $\mathrm{K} 438$. The methyl fragment of the 1-carboxyprop-1-enyloxy ligand chain is inserted into a lipophilic pocket mainly delimited by P251, L268, H334 and T361, whereas the second carboxylic group shows an ionic interaction involving K438 and it is compatible with the interaction with the Magnesium ion. Based on these considerations, we built a structurebased pharmacophore taking into account a) the $\mathrm{H}$-bond with $\mathrm{Y} 385, \mathrm{~b}$ ) the lipophilic interactions of the aromatic ring, $c$ ) the methyl fragment and d) the coordination of the carboxylic group with the Magnesium ion. The model, shown in Figure 1B, was generated using the software Ligandscout [18] and comprised (1) an $\mathrm{H}$-bond acceptor feature representing the oxygen atom of the carboxylic fragment interacting with $Y 385,(2,3)$ two hydrophobic features representing the aromatic and methyl substituents and (4) a negative feature representing the carboxylic group of the 1-carboxypropenyl-1-enyloxy fragment. In addition, the pharmacophore model was refined by adding excluded volume spheres mimicking the steric hindrance represented by the Mbtl binding site (see Experimental Section for more details). 


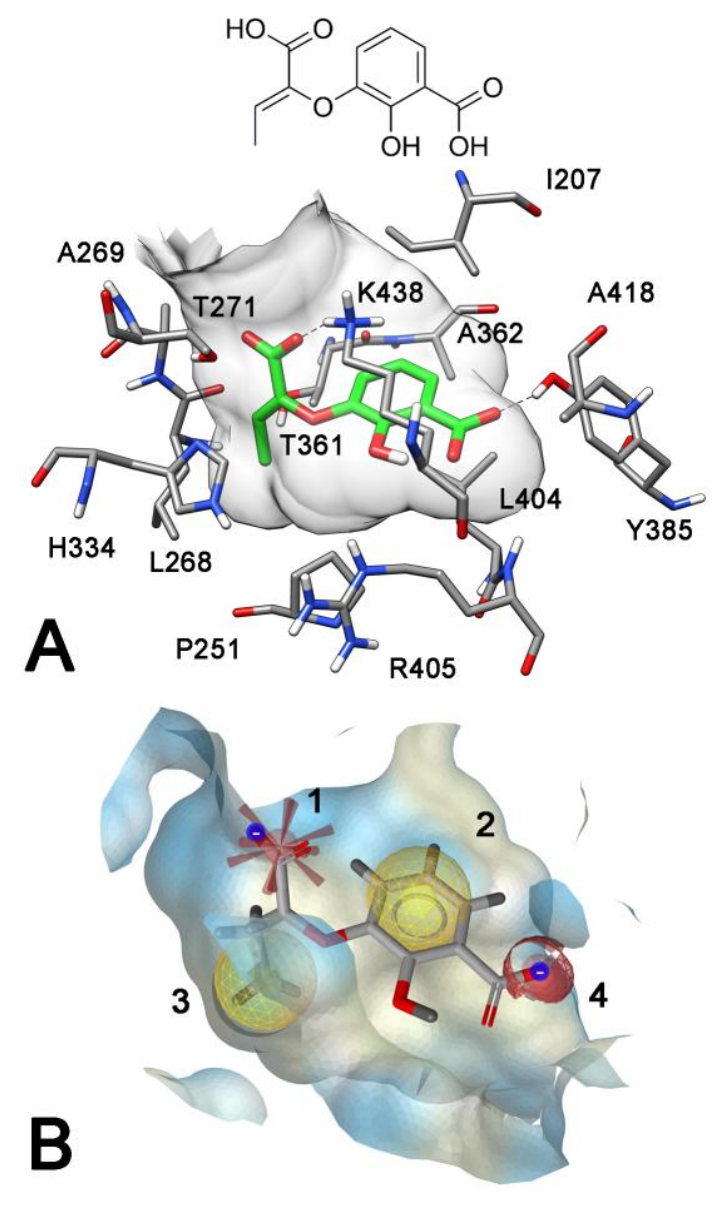

Figure 1. (A) Interactions of Methyl-AMT (green) inside the Mbtl binding site and (B) receptor-based pharmacophore model used for the VS study.

The pharmacophore model was used to screen the Enamine database, comprising about 1500000 commercially available compounds, retrieving only compounds matching all the four pharmacophore features and respecting the volume constraints given by the Mbtl binding site. By applying this strict filter, only 2050 compounds were further considered and subjected to docking studies. Recently, we have reported an evaluation of the consensus docking approach [19]. Through this approach, one ligand is docked into the target protein by means of multiple docking methods. Then, among the best-ranked poses (originated by the different docking procedures), the binding pose predicted by the largest number of docking methods is considered as the best docking pose. From a qualitative point of view, previous results highlighted that consensus docking was able to predict ligand binding poses better than the single docking evaluations [20]. Furthermore, concerning the VS studies, the results suggested that this approach performed as well as the best available methods found in the literature [20], and it was also able to experimentally identify new active molecules 
[21-23]. The 2050 compounds selected through the pharmacophore filter were subjected to a consensus docking protocol calibrated for the docking of Mbtl inhibitors. The Mtbl-tuned consensus docking protocol included 5 different docking methods, i.e. Plants and Gold with the four different fitness scoring functions, which were found to be the most reliable procedures among a total of 11 different docking methods, according to self-docking studies performed on six Mbtl-ligand co-crystal structures (see Section S1 of Supplementary material for more details). The five selected docking procedures were then applied to the 2050 commercial compounds. For each ligand, the five docking poses predicted by the five docking procedures were clustered together to search for common binding modes. The analysis revealed that only 74 compounds presented a binding pose shared by all the five docking methods and thus reached the maximum consensus level. Therefore, based on the consensus docking filter, only $3.6 \%$ of the docked compounds were considered as potential active inhibitors. These results are in agreement with our previous consensus docking calculations reported in literature, which highlighted the high filtering strictness of this procedure. In 2014, we tested the reliability of a consensus docking protocol comprising 10 different procedures by applying this technique on three different targets of the Directory of Useful Decoys (DUD) and at the end of the screening calculations only an average of $0.40 \%$ of the starting databases was considered as potentially active [19]. More recently, the whole DUD dataset has been analyzed by means of the same consensus docking approach and an average of $0.44 \%$ of the starting databases was considered as potential actives [20]. Considering that the higher the number of docking methods included in the consensus docking protocol, the higher its filtering strictness, the results obtained with the Mtbl-tuned consensus docking protocol are in line with those shown by our previous evaluations. The analysis of the binding mode predicted for the 74 potential Mtbl inhibitors identified by the consensus docking protocol revealed that only five compounds still matched the receptorbased pharmacophore model in their docking pose. On these bases, the five compounds were purchased at the highest purity level available from the commercial source ( $\geq 95 \%$ ) and subjected to biological assays to evaluate their Mbtl inhibitory activity.

Mbtl inhibitory activity. Compound effects on Mbtl enzyme activity were tested at subsaturating concentrations of substrate (chorismic acid, $\mathrm{CHA}, 50 \mu \mathrm{M}$ ), at a final concentration of $100 \mu \mathrm{M}$. The most potent Mbtl inhibitors reported in the literature belong to the class of the benzimidazole-2-thiones [24] and to the AMT-series, which is structurally related to isochorismate $[14,15]$, showing $\mathrm{IC}_{50}$ values of about $10 \mu \mathrm{M}$. In particular, MethylAMT, which emerged as the most powerful candidate of the AMT-series, was used as a 
positive control, showing an $\mathrm{IC}_{50}$ value of $11.6 \mu \mathrm{M}$, in agreement with the previously reported values [14,15]. As shown in Table 1, one compound was found to be provided with a remarkable activity (compound $\mathrm{I}, \mathrm{IC}_{50}=21.1 \mu \mathrm{M}$ ), another one displayed a modest activity (compound III, $\mathrm{IC}_{50}=77.6 \mu \mathrm{M}$ ), while the three other compounds resulted completely inactive.

Table 1. In vitro activity of compounds I-V.

Code

Compounds I and III were also subjected to molecular dynamics (MD) simulations with explicit water, in order to further analyze their predicted binding mode. Magnesium ion was also included at the entrance of the Mbtl active site in order to analyze its interaction with the ligands. Figure 2A shows the predicted binding mode of compound I into the Mbtl binding 
site. The Magnesium ion presents an octahedral coordination geometry: it is bound by a water molecule, one oxygen of the carboxylate groups of E294, E297 and E434 and the two oxygens of the carboxylate of compound I. The furan ring shows an $\mathrm{H}$-bond with $\mathrm{K} 438$, whereas the benzene ring, as for Methyl-AMT, exhibits lipophilic interactions with I207, L404 and a cation- $\pi$ interaction with K438. Finally, the nitro group strongly interacts with Y385 and, furthermore, it forms a second $\mathrm{H}$-bond with the nitrogen backbone of R405. Figure 2B shows the average minimized structure of the Mtbl-III complex. The two oxygens of the carboxyl group of the compound interact with the Magnesium ion, the benzoic acid ring shows lipophilic interactions with 1207, whereas the 2-methoxy-5-nitrophenyl fragment loses the $\mathrm{H}$-bond with the hydroxyl group of Y385 during the initial steps of the MD. However, the loss of the $\mathrm{H}$-bond with $\mathrm{Y} 385$ could be partially compensated with the presence of $\mathrm{H}$-bonds between the sulfonamide group and the backbone oxygen of T361 and the side chain of K438.

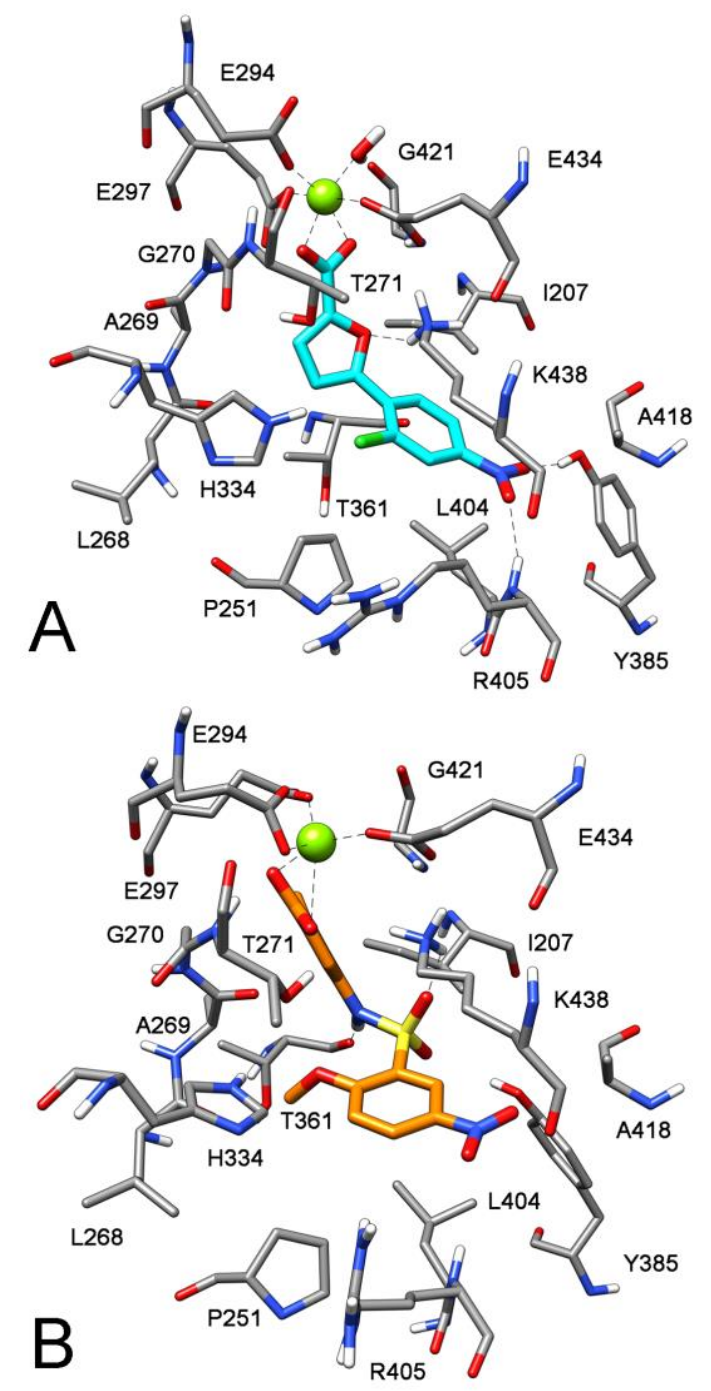

Figure 2. Minimized average structures of I (A) and III (B) docked into Mbtl binding site. 
In order to further assess the solidity of our VS workflow, a retrospective evaluation was carried out. In particular, as shown in Supplementary material (Sections S2, S3) simpler ligand-based approaches such as fingerprint similarity searches or 3D ligand similarity screenings showed a lower performance in retrieving the most active identified compound (I) from the initial commercial database. Moreover, as shown in Supplementary material (Section S4), the application of the consensus docking approach was found to outperform the single docking methods in identifying compound I from the 2050 ligands selected through the pharmacophore filter.

Structure-activity relationship (SAR) studies. In this study we considered the most active compound $\mathbf{I}$ as starting point for the development of novel Mbtl inhibitors to obtain new derivatives showing potent in vitro inhibition. In order to support the importance of each pharmacophoric feature disclosed by the modeling studies, we designed and synthesized analogs to explore the chemical space and to study the structural and functional requirements for the activity against Mbtl. We took into account two main points, namely the substitutions at the phenyl ring and the acidic moiety; the results are summarized in Table 2. By alternatively removing the chlorine atom or the para nitro group from I, we obtained the active derivative $\mathbf{1 a}$ and the inactive compound $\mathbf{1 b}$, respectively. When both these substituents were removed (1c), a complete loss of activity was detected. The replacement of the phenyl ring with pyridine (1d) afforded a partially active compound. The corresponding isomeric derivatives having the nitro substituent in meta (1e) and in ortho (1f) positions lost potency, both inhibiting Mbtl activity by $~ 50 \%$, at a concentration of $100 \mu \mathrm{M}$. The inactive compound $\mathbf{1 g}$, bearing in para position of the phenyl ring a group unable to simultaneously accept two hydrogen bonds in the suitable geometry, supported the hypothesized binding mode for this class of compounds; likewise, the substitution of the nitro group with the $\mathrm{CF}_{3}$ group (1h) led only to a retention of a modest activity (66\% residual activity). As a further attempt, the para-nitro group of $\mathbf{1 a}$ was replaced with the methylsulfonyl (1i) and sulfonamide (1j) substituents. With respect to the nitro moiety, they are larger fragments; however, they could potentially interact through two $\mathrm{H}$-bonds with the protein by means of the two oxygens, as the nitro moiety. In the first case, the replacement resulted in complete loss of activity, whereas compound $\mathbf{1 j}$ retained a low activity (84\% residual activity). The substitution of the para-nitro group with other bioisosteres led to weak $(\mathbf{1 k}, \mathbf{I})$ or inactive $(\mathbf{1} \mathbf{m}$ o) compounds. In order to assess if the carboxylic group linked to the furan conferred potency in the most active candidate 1a, the corresponding ester derivative (2a) was tested and it was found to be only slightly active ( $71 \%$ residual activity), thus confirming the 
importance of the interaction between this function and Mbtl. Finally, when the furan was substituted with a phenyl moiety (3) a partial activity was observed, while the removal of the furan led to an inactive compound (4).

Table 2. In vitro activity of compounds $\mathbf{1 a - 0 ,} \mathbf{2 a}, \mathbf{3 , 4}$.

\begin{tabular}{|c|c|c|c|}
\hline code & $\mathbf{R}_{1}$ & $\mathbf{R}_{\mathbf{2}}$ & $\begin{array}{c}\text { Residual activity at } 100 \mu \mathrm{M} \\
(\%)\end{array}$ \\
\hline $1 a$ & $4-\mathrm{NO}_{2}-\mathrm{Ph}$ & $\mathrm{H}$ & $18.2 \pm 5.1$ \\
\hline $1 \mathrm{~b}$ & 2-Cl-Ph & $\mathrm{H}$ & $92.4 \pm 13.8$ \\
\hline $1 c$ & $\mathrm{Ph}$ & $\mathrm{H}$ & $102.0 \pm 8.5$ \\
\hline 1d & & $\mathrm{H}$ & $45.0 \pm 5.1$ \\
\hline $1 e$ & $3-\mathrm{NO}_{2}-\mathrm{Ph}$ & $\mathrm{H}$ & $50.2 \pm 13.0$ \\
\hline $1 f$ & $2-\mathrm{NO}_{2}-\mathrm{Ph}$ & $\mathrm{H}$ & $55.1 \pm 22.7$ \\
\hline $1 \mathrm{~g}$ & 4-OH-Ph & $\mathrm{H}$ & $96.3 \pm 19.2$ \\
\hline $1 \mathrm{~h}$ & $4-\mathrm{Cl}$ & $\mathrm{H}$ & $66.3 \pm 9.7$ \\
\hline $1 \mathrm{i}$ & $4-\mathrm{SO}_{2} \mathrm{CH}_{3}-\mathrm{Ph}$ & $\mathrm{H}$ & $98.5 \pm 14.4$ \\
\hline $1 \mathrm{j}$ & $4-\mathrm{SO}_{2} \mathrm{NH}_{2}-\mathrm{Ph}$ & $\mathrm{H}$ & $84.6 \pm 16.2$ \\
\hline $1 k$ & & $\mathrm{H}$ & $46.8 \pm 8.3$ \\
\hline 11 & & $\mathrm{H}$ & $58.0 \pm 8.9$ \\
\hline $1 \mathrm{~m}$ & 4-CN-Ph & $\mathrm{H}$ & $125.6 \pm 17.9$ \\
\hline $1 n$ & 4-COOH-Ph & $\mathrm{H}$ & $92.4 \pm 11.8$ \\
\hline
\end{tabular}




\begin{tabular}{|l|l|l|l|}
\hline 10 & & & $104.4 \pm 7.8$ \\
\hline $2 a$ & & \\
3 &
\end{tabular}

The SAR studies allowed to evidence that compound 1a shows an improved potency with respect to our initial hit $\mathrm{I}$, as it displayed an $\mathrm{IC}_{50}$ value of $7.6 \pm 1.6 \mu \mathrm{M}$ (Figure 3A). Moreover, the Mbtl kinetic analysis in the presence of 1a proved that this compound is a potent competitive inhibitor towards the chorismate binding site, displaying a $K_{i}$ value of $5.3 \pm 0.6$ $\mu \mathrm{M}$ (Figure 3B and 3C).
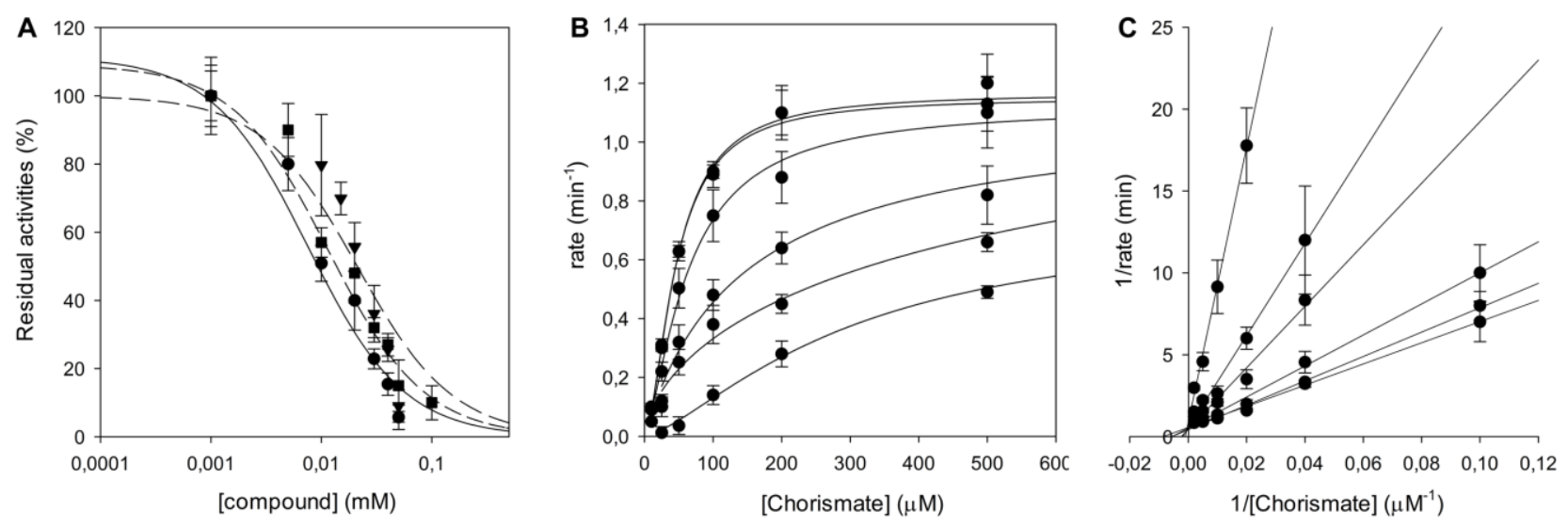

Figure 3. Effects of the compounds against Mbtl enzyme activity. (A) Graph comparing $\mathbf{I}(\mathbf{\nabla})$ and MethylAMT ( $\mathbf{\square})$ with $1 \mathrm{a}(\bullet)$. IC $\mathrm{I}_{50}$ values were determined at subsaturating concentrations of $\mathrm{CHA}(50 \mu \mathrm{M})$. (B) Steady state kinetics analysis towards $\mathrm{CHA}$ of Mbtl in the presence of different concentrations of $\mathbf{1 a}(0,5,10,20,50$ and $100 \mu \mathrm{M}$ ) highlights the competitive behavior of the inhibitor. (C) Global reciprocal plot of data in panel B. All data are mean SD of three replicates. 
Mtb minimal inhibitory concentration determination $\left(\mathrm{MIC}^{99}\right)$. In order to assess its potency, the inhibitor 1a was investigated for its antibacterial activity against $M$. tuberculosis $\mathrm{H} 37 \mathrm{Rv}$, together with $\mathbf{2 a}$, on the basis of the higher activity of esters with respect to the carboxylic acids on this parasite, probably owing to a better ability to cross the mycobacterial cell wall [15]. Compound 1a showed a $\mathrm{MIC}^{99}$ of $156 \mu \mathrm{M}$, whereas for $2 \mathrm{a}$ we did not detect an improvement of the cellular potency, probably due to its low solubility in the assay medium. It is noteworthy that, to the best of our knowledge, 1a can be considered as one of the Mbtl inhibitors showing the best antimycobacterial activity.

Mycobactin biosynthesis inhibition. To assess if the antitubercular activity is indeed related with iron-uptake inhibition, we used the non-pathogenic M. bovis BCG, whose siderophores closely resemble the structure of $M t b$ mycobactins [26,27]. The compound, when assayed against cells grown in the low iron containing Chelated Sauton's medium, showed a MIC value 3-fold lower than against mycobacteria grown in 7H9 medium (80 $\mu \mathrm{M}$ vs $240 \mu \mathrm{M}$ ), suggesting that the mechanism of action of 1a involves iron uptake. Thus, in order to support the correlation of the antitubercular activity of 1a with Mbtl inhibition, the effects of the compound on siderophore production were assessed in M. bovis BCG cells, grown in iron-depleted medium and in the presence of different sub-lethal concentrations of compound 1a. To this purpose, the levels of siderophore production were measured using Universal CAS liquid assay [28] and through the mycobactins isolation and quantification. As shown in Figure S94, the CAS assay performed on the medium of the cells grown in different concentrations of $1 \mathbf{a}$, showed a removal of iron inversely related to the concentration of the compound. Similarly, the concentration of mycobactins, determined in the same cells, decreased at higher concentrations of 1a, confirming that the inhibitory effect towards Mtb growth could be due to mycobactin biosynthesis inhibition.

Chemistry. Compounds 1a,e and 4 were purchased and their purity was assessed using HPLC ( $\geq 95 \%$ ). Details are provided in Supplementary material. The methyl ester (2a) of 1a, as well as compounds $\mathbf{5}$ and $\mathbf{6}$, were obtained according to a common procedure (FischerSpeier esterification). Compounds $\mathbf{1} \mathbf{b}-\mathbf{d}, \mathbf{f}-\mathbf{0}, \mathbf{3}$ were synthesized by a Suzuki-Miyaura coupling reaction [29], followed by a base-catalyzed hydrolysis of the ester function. The synthetic pathways are reported in the Scheme 2, the general procedures are described in the experimental section, while all the details concerning the specific synthetic steps and the analytical data are provided in Supplementary material. 

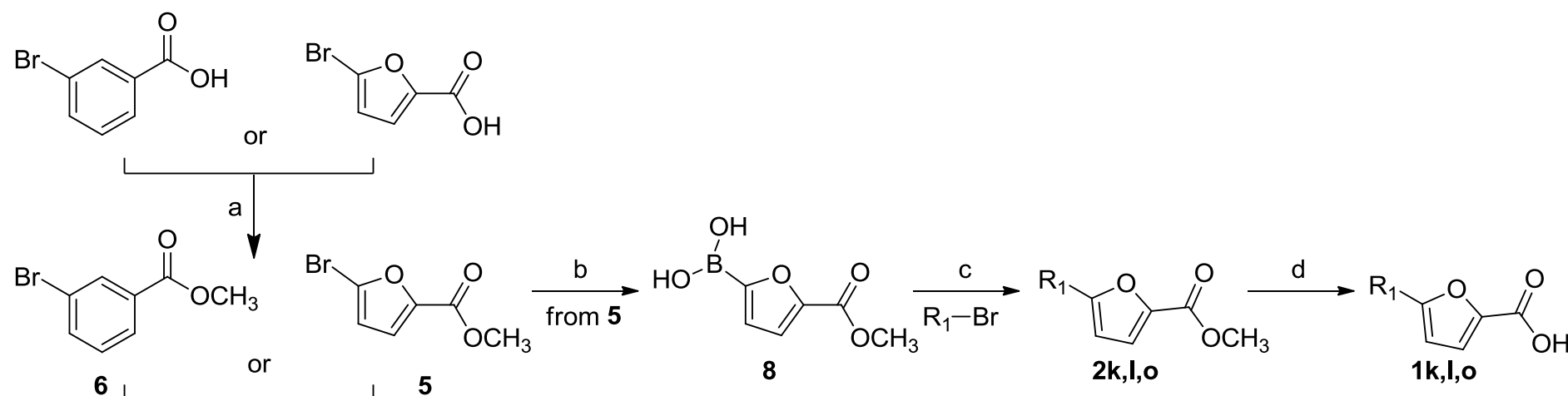$$
\text { c } \downarrow \underset{\substack{\mathrm{B}^{-} \\ \mathrm{O}}}{\mathrm{R}_{1}{ }^{-\mathrm{OH}}}
$$

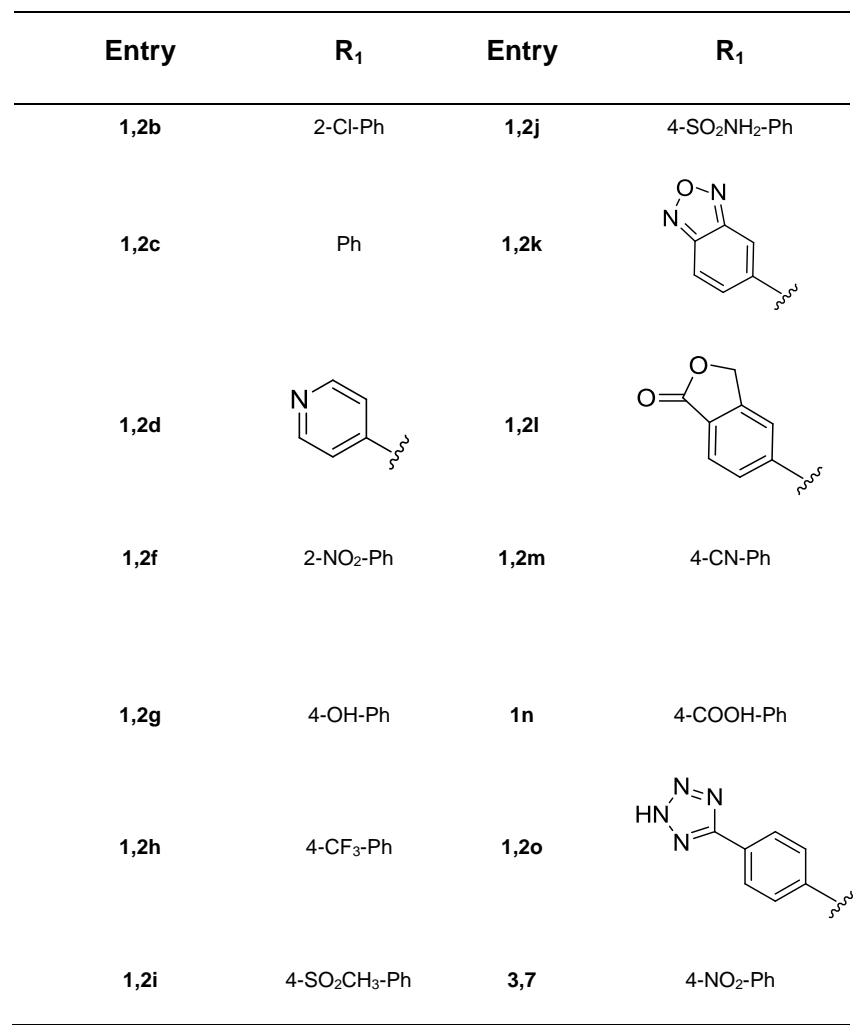

Scheme 2. Reagents and conditions: (a) conc. $\mathrm{H}_{2} \mathrm{SO}_{4}, \mathrm{CH}_{3} \mathrm{OH}$, reflux, $24 \mathrm{~h}$; (b) i-PrMgCl, Bis[2-(N,Ndimethylamino)ethyl]ether, $\mathrm{B}\left(\mathrm{OCH}_{3}\right)_{3}, \mathrm{THF}, 15^{\circ} \mathrm{C} 20 \mathrm{~min} \rightarrow 20^{\circ} \mathrm{C}, 30 \mathrm{~min} \rightarrow 0^{\circ} \mathrm{C}, 10 \mathrm{~min}, \mathrm{~N}_{2}$; (c) appropriate reagent, $\mathrm{Pd}\left(\mathrm{PPh}_{3}\right)_{2} \mathrm{Cl}_{2}, 2 \mathrm{M} \mathrm{Na} \mathrm{CO}_{3}, 1,4$-dioxane, $90^{\circ} \mathrm{C}$, overnight, $\mathrm{N}_{2}$, (for $2 \mathbf{k}$,l,o $60^{\circ} \mathrm{C}, 1$ h, M.W.); (d) $1 \mathrm{M}$ $\mathrm{NaOH}, \mathrm{CH}_{3} \mathrm{CH}_{2} \mathrm{OH} / \mathrm{THF}$ 1:1, reflux, $5 \mathrm{~h}$ (for 1d: $\mathrm{LiOH} \cdot \mathrm{H}_{2} \mathrm{O} \mathrm{THF} / \mathrm{H}_{2} \mathrm{O}, 20^{\circ} \mathrm{C}, 2 \mathrm{~h}$; for 1l: $0.5 \mathrm{M} \mathrm{NaOH}$, $\mathrm{CH}_{3} \mathrm{CH}_{2} \mathrm{OH} / \mathrm{THF} 1: 1,20^{\circ} \mathrm{C}, 30 \mathrm{~min}$ ); (e) $\mathrm{KOH}, \mathrm{H}_{2} \mathrm{O}$, reflux, $6 \mathrm{~h}$.

\section{CONCLUSIONS}

Mbtl is a validated and attractive target for the treatment of tuberculosis, and its inhibition leads to a reduced mycobacterial pathogenicity without toxicity issues, due to the absence of this target in the host. Starting from the computational analysis of the interaction between 
Mbtl and methyl-AMT, a VS study was carried out, identifying the hit compounds I and III as interesting inhibitors. Considering the most active compound $\mathbf{I}$, we described the synthesis and biological evaluation of new Mbtl inhibitors, based on the furan scaffold and having different substituents on the phenyl ring. Initial SAR studies applied on I allowed the identification of a potent competitive Mbtl inhibitor, compound 1a. Remarkably, 1a exerts a slightly better in vitro inhibitory activity $\left(\mathrm{IC}_{50}=7.6 \mu \mathrm{M}\right)$ compared to Methyl-AMT $\left(\mathrm{IC}_{50}=11.6\right.$ $\mu \mathrm{M})$, which is the best Mbtl inhibitor discovered to date. In addition, 1a displayed a MIC ${ }^{99}$ value of $156 \mu \mathrm{M}$, currently being the first potent Mbtl inhibitor endowed with a promising antitubercular activity. In addition, siderophore assay suggested that the inhibitory effect of 1a towards Mtb growth could be due to mycobactin biosynthesis inhibition, thus highlighting the importance of this pathway as target for the development of therapeutic interventions. It is noteworthy that compound 1a contains a nitrophenyl moiety as a bioisoster of the salicylic moiety of Methyl-AMT. Even if the nitro group is a cause for concern in medicinal chemistry, for the first time we demonstrated the effective replacement of one carboxylic group of Methyl-AMT without loss of Mbtl inhibitory potency, allowing, at the same time, a better permeability through the Mtb cell wall. Our results are the starting point to expand the SAR study among this class of derivatives, exploiting other hitherto unconsidered pharmacophoric features, with the aim of disclosing new lead compounds lacking the nitro group, making our series more attractive for further developments.

In conclusion, the analysis of the hypothetical binding mode of this class of compounds suggested potential points of modification that led to significant developments, and that could be further explored to increase the potency of these derivatives.

\section{EXPERIMENTAL SECTION:}

Pharmacophore model generation. One of the main drawbacks of the VS consensus docking approach is the required computing time, because by using this method a whole data set of molecules should be subjected to different docking procedures [19]. Therefore, in order to reduce the number of compounds to be analyzed, a pharmacophore screening was used as a prefilter. The pharmacophore hypothesis was built on the basis of the $3 \mathrm{D}$ structure of Mbtl co-crystallized with methyl-AMT (PDB code 3VEH) [17]. The software LigandScout 4.08 [18] was used for the creation of the receptor-based pharmacophore models. An exhaustive pharmacophore model including all the possible features identified 
by the program was generated and, subsequently, only the desired features were retained in the final pharmacophore model, for a total of four features. The excluded volume spheres were defined on the basis of the receptor structure as implemented in the default LigandScout configuration.

Database Generation and Pharmacophore Screening. The Enamine database, comprising about 1500000 commercially available compounds was used as the screening database. The idbgen-gui utility of LigandScout was employed to create the LigandScout 3D database. The iCon Best conformational sampling method was applied. The 3D database was then screened using the previously created receptor-based pharmacophore model and the receptor excluded volume, imposing that only the compounds matching all the four pharmacophoric features of the model were retrieved.

Docking procedures and pose filtering. Prior to the docking analysis, a self-docking evaluation of the main Mbtl-inhibitor X-ray complexes (PDB codes 3RV6, 3RV7, 3RV8, 3RV9, 3ST6 and 3VEH) was carried out using Autodock, Autodock vina, Dock, Fred, Gold with the four fitness functions implemented (i.e., GoldScore, ChemScore, Astex Statistical Potential and ChemPLP), Glide with the standard and extra precision methods and Plants. The use of Gold, with the four different fitness functions, and Plants resulted to be the most reliable ones as they showed an average RMSD lower than $2.0 \AA$. For all the docking procedures the region of interest for the docking studies was defined in such a manner that it contained all residues that stayed within $10 \AA$ from the ligand in the $\mathrm{X}$-ray structure.

Gold. The "allow early termination" command was deactivated, while the possibility for the ligand to flip ring corners was activated. For all other parameters, Gold defaults were used and the ligands were subjected to 30 genetic algorithm runs. Four docking analyses were carried out, corresponding to the four implemented fitness functions.

Plants. This docking software uses Ant Colony Optimization, a state-of the-art global optimization algorithm to find minima of a scoring function representing favorable complex structures [30,31]. ChemPLP scoring function was employed to score protein-ligand interactions as well as intra-ligand clash terms. Standard settings for all parameters were used for the scoring function and the optimization algorithm (search speed setting: "speed1").

Consensus docking evaluation. By applying the five docking methods, five different binding dispositions (best-scored docking pose) resulted from the docking of each ligand into each 
protein binding site. The RMSD of these docking poses against the remaining four was evaluated by using the rms_analysis software of the Gold suite. On this basis, for each ligand docked into the protein binding site, a $5 \times 5$ matrix was generated reporting the RMSD results. By using an in-house program [18], these results were clustered, so that among the five results, all of the similar docking poses were clustered together. As a clustering algorithm, we used the complete-linkage method, which is an agglomerative type of hierarchical clustering. We selected an RMSD clustering threshold of $2.0 \AA$; therefore, the so-obtained clusters contained the group of poses that were less than $2.0 \AA$ away from all others poses belonging to the same cluster. All of the ligands showing a consensus level of five were taken into account.

Filtering of docking results. The filtering of the docking results was carried out by superimposing the docked compounds to the pharmacophore directly from the supplied poses, without changing their coordinates. The retrieval of compounds matching all the four pharmacophoric features of the model was imposed in this search.

MD simulations. All simulations were carried out using AMBER 14 [32]. MD simulations were performed using the ff14SB force field at $300 \mathrm{~K}$. The complex was placed in a rectangular parallelepiped water box. Magnesium ion was inserted analyzing its disposition and interaction into the 2FN1 PDB code [33]. Many force field-based MD simulations of biological systems containing $\mathrm{Mg}^{2+}$ have already been reported in literature [34-37]. In the present study we used the particle mesh Ewald (PME) compatible Lennard-Jones parameters for divalent metal ions in explicit solvent reported by Merz and co-workers [38]. An explicit solvent model for water, TIP3P, was used, and the complexes were solvated with a $20 \AA$ water cap. Sodium ions were added as counterions to neutralize the system. Prior to MD simulations, two steps of minimization were carried out; in the first stage, we kept the protein fixed with a position restraint of $500 \mathrm{kcal} / \mathrm{mol} \cdot \AA^{2}$ and we solely minimized the positions of the water molecules. In the second stage, we minimized the entire system through 5000 steps of steepest descent followed by conjugate gradient (CG) until a convergence of $0.05 \mathrm{kcal} / \AA \cdot \mathrm{mol}$, using the same procedure described above. PME electrostatics and periodic boundary conditions were used in the simulation [39]. The MD trajectory was run using the minimized structure as the starting conformation. The time step of the simulations was 2.0 fs with a cutoff of $10 \AA$ for the non-bonded interaction, and SHAKE was employed to keep all bonds involving hydrogen atoms rigid. Constant-volume periodic boundary MD was carried out for $0.5 \mathrm{~ns}$, during which the temperature was raised from 0 to 
$300 \mathrm{~K}$. Then $19.5 \mathrm{~ns}$ of constant pressure periodic boundary MD was performed at $300 \mathrm{~K}$ using the Langevin thermostat to maintain constant the temperature of our system. All the $\alpha$ carbons of the protein were blocked with a harmonic force constant of $10 \mathrm{kcal} / \mathrm{mol} \cdot \AA^{2}$ for the first 3.5 ns while in the last 16.5 ns no constraints were applied. General Amber force field (GAFF) parameters were assigned to the ligands, while partial charges were calculated using the AM1-BCC method as implemented in the Antechamber suite of AMBER 14.

General Synthetic Procedures and Materials. Commercial products (1a,e and 4), chemicals and solvents were of reagent grade; they were purchased from suppliers (SigmaAldrich) and used as received. Anhydrous solvents were utilized without further drying. Aluminum backed Silica Gel 60 plates $(0.2 \mathrm{~mm}$, Merck) were used for analytical TLC, to follow the course of the reaction. Silica gel 60 (Merck 40-63 $\mu \mathrm{m}$ ) was used for the purification of intermediates and final compounds, through flash column chromatography. Melting points were determined in open capillary tubes with a Büchi Melting Point 510. All tested compounds were characterized by the means of FT-IR, ${ }^{1} \mathrm{H}$ NMR, ${ }^{13} \mathrm{C}$ NMR, MS and HPLC to check their purity. ${ }^{1} \mathrm{H}$ and ${ }^{13} \mathrm{C}$ NMR spectra were acquired at ambient temperature with a Varian-Oxford $300 \mathrm{MHz}$ instrument, operating at $300 \mathrm{MHz}$ for ${ }^{1} \mathrm{H}$ and $75 \mathrm{MHz}$ for ${ }^{13} \mathrm{C}$. Chemical shifts are expressed in ppm $(\delta)$ from tetramethylsilane resonance in the indicated solvent (TMS: $\delta=0.0 \mathrm{ppm}$ ), while $J$-couplings are given in Hertz. The APT sequence was used when deemed necessary. IR spectra were acquired with a Perkin Elmer Spectrum One FT-IR in a spectral region between 4000 and $450 \mathrm{~cm}^{-1}$ and analyzed by transmittance technique with 32 scansions and $4 \mathrm{~cm}^{-1}$ resolution. Solid samples were mixed in a mortar with $\operatorname{KBr}(1: 100)$ and pressed, using a hydraulic press (14 tons), to small tablets. MS analyses were carried out with a Thermo Finnigan (MA, USA) LCQ Advantage system, equipped with a quaternary pump, a Diode Array Detector (working wavelength: $254 \mathrm{~nm}$ ) and a MS spectrometer, with an Electrospray ionization source and an Ion Trap mass analyzer (ionization: ESI positive or ESI negative; capillary temperature: $250^{\circ} \mathrm{C}$; source voltage: $5.50 \mathrm{kV}$; source current: $4.00 \mu \mathrm{A}$; multipole 1 and 2 offset: $-5.50 \mathrm{~V}$ and $-7.50 \mathrm{~V}$, respectively; intermultipole lens voltage: $-16.00 \mathrm{~V}$; trap $\mathrm{DC}$ offset voltage: $-10.00 \mathrm{~V}$ ). The purity of the compounds was assessed by means of HPLC $(\lambda=220 \mathrm{~nm})$ and was $\geq 95 \%$ unless otherwise stated. Column: EVO C18 Phenomenex. Eluent system: aqueous formic acid (0.05\%) / acetonitrile 50:50. Total flow: $1.2 \mathrm{~mL} / \mathrm{min}$. Injection volume: $30 \mu \mathrm{L}$.

Compounds I-V were purchased from Enamine at the highest purity level available $(\geq 95$ $\%)$. 
Details about specific synthetic procedures and analytical data (Table S2) of compounds $\mathbf{1 a - 0 ,} \mathbf{2 a}, \mathbf{3}$ and $\mathbf{4}$ are reported in Supplementary material.

\section{Procedure A for the synthesis of compounds $2 b-d, f-j, m$ and 7.}

Methyl 5-bromofuran-2-carboxylate $(5,1 \mathrm{mmol})$, the appropriate phenylboronic acid (1.3 $\mathrm{mmol})$ and bis(triphenylphosphine)palladium(II) dichloride $(5 \% \mathrm{~mol})$ were dissolved in dry 1,4-dioxane $(10 \mathrm{~mL})$, under nitrogen atmosphere. A 2M sodium carbonate solution (2 mmol) was then added and the resulting mixture was stirred overnight at $90^{\circ} \mathrm{C}$. After completion, the solution was cooled to room temperature and then filtered on a celite pad. The filtrate was diluted with water and extracted with ethyl acetate $(3 \times 4 \mathrm{~mL})$. The organic layer was dried over anhydrous sodium sulfate, filtered and concentrated in vacuo. Compound 7 was obtained from methyl 3-bromobenzoate $(6,1 \mathrm{mmol})$ and 4-nitrophenylboronic acid (1.3 mmol) following the same procedure.

\section{Procedure B for the synthesis of compounds $2 k, l, 0$.}

Methyl 5-boronofuran-2-carboxylate $(\mathbf{8}, 1.3 \mathrm{mmol})$, the appropriate bromo derivatives (10, or 12 , or $13,1.0 \mathrm{mmol}$ ) and bis(triphenylphosphine)palladium(II) dichloride (5\% mol) were dissolved in dry 1,4-dioxane $(10 \mathrm{~mL})$, under nitrogen atmosphere. A $2 \mathrm{M}$ sodium carbonate solution $(2 \mathrm{mmol})$ was then added and the resulting mixture was stirred in a microwave synthesizer (Biotage ${ }^{\circledR}$ Initiator Classic) for $1 \mathrm{~h}$ at $60^{\circ} \mathrm{C}$. After completion, the solution was cooled to room temperature and then filtered on a celite pad. The filtrate was diluted with water and extracted with ethyl acetate $(3 \times 4 \mathrm{~mL})$. The organic layer was dried over anhydrous sodium sulfate, filtered and concentrated in vacuo.

\section{Procedure $C$ for the synthesis of compounds $1 b-d, f-o$ and 3.}

The appropriate methyl ester derivative (2b-d, $\mathbf{f}-\mathbf{m}, \mathbf{0}, \mathbf{7}, 1 \mathrm{mmol})$ was dissolved in a mixture of tetrahydrofuran - ethanol 1:1 $(15 \mathrm{~mL})$ and a $1 \mathrm{M}$ solution of sodium hydroxide (2.5 $\mathrm{mmol}$ ) was added dropwise while stirring. The reaction mixture was heated at reflux for $5 \mathrm{~h}$. After completion, the solvent was evaporated under reduced pressure; the aqueous phase was washed with chloroform $(1 \times 5 \mathrm{~mL})$, acidified with hydrochloric acid $(3 \mathrm{M})$ and then extracted with ethyl acetate $(3 \times 7 \mathrm{~mL})$. The organic layers were washed with brine, dried over anhydrous sodium sulfate and then concentrated in vacuo. The resulting solid was finally washed with cool hexane $(3 \mathrm{~mL})$. Variations to this general procedure were applied to the synthesis of $\mathbf{1 d} \mathbf{d} \mathbf{I}$ (see Supplementary material for details). 


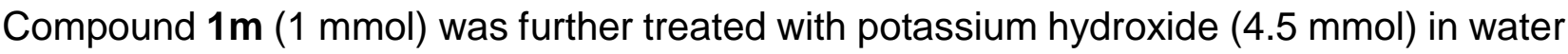
$(12 \mathrm{~mL})$ at reflux for $6 \mathrm{~h}$. After completion, the solution was acidified with hydrochloric acid $(10 \mathrm{M})$ and then extracted with ethyl acetate $(3 \times 7 \mathrm{~mL})$. The organic layers were washed with brine, dried over anhydrous sodium sulfate and then concentrated in vacuo to obtain 1n as a light yellow solid.

Characterization of 1a-0, 2a, 3, 4. All compounds were characterized using ${ }^{1} \mathrm{H} N M R,{ }^{13} \mathrm{C}$ NMR, MS, IR spectroscopy, and RP-HPLC. The purity was $\geq 95 \%$ according to analytical RP-HPLC. ${ }^{1} \mathrm{H}$ NMR and ${ }^{13} \mathrm{C}$ NMR spectra, together with RP-HPLC chromatograms are provided in Supplementary material.

Production and purification of chorismic acid. Chorismic acid was produced using the Escherichia coli KA12 strain, according to Grisostomi et al. [40] and purified by a modified protocol described in Supplementary material.

Mbtl Expression, Purification and Inhibition assays. Rv2386c gene, encoding the Mbtl was cloned into pET28b expression vector, and the protein was expressed in BL21 (DE3) E. coli cells and purified as reported in Supplementary Information. Enzymatic activity was determined at $37^{\circ} \mathrm{C}$ by L-lactic coupled assay, as previously described [14]. The standard reaction mixture contained 50 mM HEPES pH 8.0, $0.2 \mathrm{mM} \mathrm{NADH}, 10 \mathrm{mM} \mathrm{MgCl}_{2}, 3$ units of L-lactic dehydrogenase (Sigma Aldrich), 10-20 $\mu \mathrm{M} \mathrm{Mbtl,} \mathrm{and} \mathrm{the} \mathrm{reactions} \mathrm{were} \mathrm{started} \mathrm{by}$ the addition of the substrate (CHA). Steady-state kinetic parameters were determined by assaying the enzyme at variable concentrations of chorismic acid (10-500 $\mu \mathrm{M})$. The experiments were performed in triplicate, and the kinetic constants $K_{m}$ and $K_{c a t}$ determined by fitting the data to the Michaelis-Menten equation using Origin 8 software. Initially, Mbtl inhibition was screened for all compounds at $100 \mu \mathrm{M}$ (dissolved in a solution containing $10 \%$ DMSO) and $50 \mu \mathrm{M}$ chorismic acid. The compound solubility in the medium assay was verified up to $100 \mu \mathrm{M}$ concentration. For compounds that significantly inhibited the enzyme activity, $\mathrm{IC}_{50}$ and $K_{\mathrm{i}}$ values were determined. For $\mathrm{IC}_{50}$ determinations, the enzyme activities were measured in the presence of compound and values were estimated according to the Equation 1, where $A_{[l]}$ is the enzyme activity at inhibitor concentration [I] and $A_{[0]}$ is the enzyme activity without inhibitor.

$$
A_{[l]}=A_{[0]} \times\left(1-\frac{[l]}{[I]+I C_{50}}\right) \quad \text { Equation } 1
$$


The inhibition constant $\left(K_{i}\right)$ values were determined by assaying the Mbtl enzymatic activity at different substrate and compound concentrations, using an adapted equation for competitive inhibition (Equation 2) with Origin 8 software.

$$
v=\frac{V_{\max }[\mathrm{S}]}{[\mathrm{S}]+K_{\mathrm{m}}\left(1+\frac{[I]}{K_{\mathrm{i}}}\right)} \quad \text { Equation } 2
$$

Pan Assay Interference Compounds (PAINS) analysis. As reported by Baell and Holloway, there is a number of substructural features which could help to identify compounds that appear as PAINS in many biochemical high-throughput screens $[41,42]$. The corresponding filters have been included in the Filter-it ${ }^{\mathrm{TM}}$ software and compound $\mathbf{1 a}$ was thus filtered by using this program. The result highlighted that this compound did not possess any of the substructural features shared by the most common PAINS. Moreover, to verify the possible formation of aggregates, the ability of compound $\mathbf{1 a}$ to inhibit Mbtl activity was also tested in the presence of $0.1 \mathrm{mg} / \mathrm{mL}$ of bovine serum albumin (BSA) or in the presence of $0.01 \%(\mathrm{v} / \mathrm{v})$ Triton $\mathrm{X}-100$ as detergent [43]. As reported in Figure S93, the $\mathrm{IC}_{50}$ value in the presence of BSA $(7.28 \pm 1.41 \mu \mathrm{M})$ or in the presence of Triton X-100 (8.00 $\pm 1.28 \mu \mathrm{M}$ ) are not significantly different, supporting the notion that the ligand did not act as an aggregate. Finally, to exclude a promiscuous enzyme inhibition due to covalent reaction with cysteines [44]. Mbtl inhibition activity of compound $\mathbf{1 a}$ was also tested in the presence of $100 \mu \mathrm{M}$ of 1,4-dithio-DL-threitol (DTT). As depicted in Figure S93, the IC50 was not influenced by DTT $(7.84 \pm 1.07 \mu \mathrm{M})$, thus excluding an interaction of $1 \mathrm{a}$ with the Mbtl cysteines.

Minimal inhibitory concentration determinations. The $\mathrm{MIC}^{99}$ of $\mathbf{1 a}$ and $\mathbf{2 a}$, against $M$. tuberculosis H37Rv, was determined in solid medium. A single colony was inoculated in complete Middlebrook 7H9 supplemented with 10\% OADC Middlebrook Enrichment, and grown at $37{ }^{\circ} \mathrm{C}$ until exponential growth phase $\left(\sim 10^{8} \mathrm{CFU} / \mathrm{mL}\right)$. Dilutions to the final concentration of $\sim 10^{6} \mathrm{CFU} / \mathrm{mL}$ were performed and about $1 \mu \mathrm{L}$ of cell culture was streaked onto plates containing two-fold serial dilutions of appropriate compound. MIC values were assigned as the lowest drug concentrations inhibiting bacterial cell growth. The experiments were performed in triplicate. Additionally, the compound was assayed against $M$. bovis BCG in liquid medium, in high and low iron media, by determining the MIC against cells grown both in 7H9/OADC medium and in low-iron Chelated Sauton's medium [45].

Siderophore production assay. Siderophore activity present in the culture was tested in M. bovis BCG through the isolation of mycobactins [46] or using the Universal CAS liquid 
assay [28]. To this purpose, M. bovis was grown in 7H9 medium, subcultured in chelated Sauton's medium, then diluted $1: 1000$ in chelated Sauton's containing different concentrations of compound 1a. After 15 days of incubation at $37^{\circ} \mathrm{C}$, cells were harvested by centrifugation. For the isolation of mycobactins, cell pellets were extracted in ethanol overnight. $0.1 \mathrm{M} \mathrm{FeCl}_{3}$ in ethanol was added until no further change in colour was observed, and the mixture incubated at room temperature for $1 \mathrm{~h}$. Mycobactins were then extracted in chloroform, washed with water 3 times to remove the excess of iron, and evaporated. Residue was dissolved in methanol. The concentration of mycobactins was determined by measuring the absorbance at $450 \mathrm{~nm}$ (1\% solution of mycobactins gives an absorbance of 42.8) [46]. For Universal CAS assay $100 \mu$ of cell cultures supernatant was mixed with 100 $\mu \mathrm{l}$ of CAS assay liquid solution in 96 well plate, incubated $10 \mathrm{~min}$ at room temperature, then the absorbance was measured at $630 \mathrm{~nm}$. The siderophore units were calculated using the following equation

$$
\frac{A_{r}-A_{s}}{A_{r}} \times 100 \text { Equation } 3
$$

where $A_{r}$ is the absorbance at $630 \mathrm{~nm}$ of the blank medium with CAS assay solution and $A_{s}$ is the absorbance of the culture supernatants with CAS assay solution.

\section{AUTHOR INFORMATION}

\section{Corresponding Authors}

*stefania.villa@unimi.it. ++390250319368, ORCID: 0000-0002-0636-7589;

*tiziano.tuccinardi@unipi.it. ++390502219595, ORCID: 0000-0002-6205-4069.

\section{Author Contributions.}

¥These authors contributed equally.

\section{Notes}

The authors declare no competing financial interest.

\section{Acknowledgments}

We acknowledge the University of Milan (Linea B) and the University of Pavia for the financial support. We thank Prof. Peter Kast, ETH Zurich, for kindly providing the E. coli KA12 strain. 
Supplementary material. Virtual screening evaluation, NMR spectra, IR and MS data, HPLC chromatograms, chemical and biological experimental procedures and supplementary figures, PDB file of the I-Mbtl complex. This material is available free of charge at website.

\section{ABBREVIATIONS USED}

Mtb, Mycobacterium tuberculosis; Mbtl, Mycobacterium tuberculosis salicylate synthase; TB, Tuberculosis; VS, virtual screening; DUD, Directory of Useful Decoys; CHA, chorismic acid; CG, conjugate gradient; PME, Particle mesh Ewald; GAFF, general Amber force field; BSA, bovine serum albumin; CAS, chrome azurol S.

\section{REFERENCES}

[1] Global tuberculosis report 2017. Geneva: World Health Organization; 2017. Licence: CC BY-NC-SA 3.0 IGO

[2] D.A. Mitchison, The diagnosis and therapy of tuberculosis during the past 100 years. Am. J. Respir. Crit. Care Med. 171, (2005) 699-706.

[3] D.G. Russell, C.E. Barry III, J.L. Flynn, Tuberculosis: what we don't know can, and does, hurt us, Science 328, (2010) 852-856.

[4] A. Campaniço, R. Moreira, F. Lopes, Drug discovery in tuberculosis. New drug targets and antimycobacterial agents, Eur. J. Med. Chem. 150 (2018) 525e545.

[5] V. Singh Bais, B. Mohapatra, N. Ahamad, S. Boggaram, S. Verma, B. Prakash Investigating the inhibitory potential of 2-Aminopurine metal complexes against serine/threonine protein kinases from Mycobacterium tuberculosis, Tuberculosis, 108, (2018) 47-55

[6] F. Meneghetti, S. Villa, A. Gelain, D. Barlocco, L.R. Chiarelli, M.R. Pasca, L. Costantino, Iron acquisition pathways as targets for antitubercular drugs. Curr. Med. Chem. 23, (2016) 4009-4026.

[7] L. Fanzani, F. Porta, S. Villa, A. Gelain, A.P. Lucarelli, E. Parisini, Mycobacterium tuberculosis Low Molecular Weight Phosphatases (MPtpA and MPtpB): From Biological Insight to Inhibitors, Curr. Med. Chem. 22, (2015) 3110-3132.

[8] K.S. Singh, R. Sharma, D. Keshari, N. Singh, S.K. Singh, Down-regulation of malate synthase in Mycobacterium tuberculosis $\mathrm{H} 37 \mathrm{Ra}$ leads to reduced stress tolerance, persistence and survival in macrophages, Tuberculosis 106, (2017) 73-81.

[9] S.E. Borisov, K. Dheda, M. Enwerem, R. Romero Leyet, L. D'Ambrosio, R. Centis, G. Sotgiu, S. Tiberi, J.W. Alffenaar, A. Maryandyshev, E. Belilovski, S. Ganatra, A. Skrahina, O. Akkerman, A. Aleksa, R. Amale, J. Artsukevich, J. Bruchfeld, J. A. Caminero, I. Carpena Martinez, L. Codecasa, M. Dalcolmo, J. Denholm, P. Douglas, R. Duarte, A. Esmail, M. Fadul, A. Filippov, L. Davies Forsman, M. Gaga, J.A. Garcia-Fuertes, J.M. García-García, G. Gualano, J. Jonsson, H. Kunst, J.S. Lau, B. Lazaro Mastrapa, J.L.Teran Troya, S. Manga, K. Manika, P. González Montaner, J. Mullerpattan, S. Oelofse, M. Ortelli, D.J. Palmero, F. Palmieri, A. Papalia, A. Papavasileiou, M. C. Payen, E. Pontali, C. Robalo Cordeiro, L. Saderi, T.D. Sadutshang, T. Sanukevich, V. Solodovnikova, A. Spanevello, S.Topgyal, F.Toscanini, A.R. Tramontana, Z.F. Udwadia, P. Viggiani, V. White, A. Zumla, G.B. Migliori, Effectiveness and safety of bedaquiline-containing regimens in the treatment of MDR- and XDR-TB: a multicentre study. Eur. Respir. J. 49, (2017) 1700387.

[10] A. Zumla, P. Nahid, S.T. Cole, Advances in the development of new tuberculosis drugs and treatment regimens. Nat. Rev. Drug Discov. 12, (2013) 388-404.

[11] K.N. Raymond, E.A. Dertz, S.S. Kim, Enterobactin: an archetype for microbial iron transport. Proc. Natl. Acad. Sci. U. S. A. 100, (2003) 3584-3588.

[12] J.J. De Voss, K. Rutter, B.G. Schroeder, H. Su, Y. Zhu, C.E. Barry III, The salicylate-derived mycobactin siderophores of Mycobacterium tuberculosis are essential for growth in macrophages. Proc. Natl. Acad. Sci. U. S. A. 97, (2000) 1252-1257.

[13] J. Zwahlen, S. Kolappan, R. Zhou, C. Kisker, P.J. Tonge, Structure and mechanism of Mbtl, the salicylate synthase from Mycobacterium tuberculosis. Biochemistry 46, (2007), 954-964. 
[14] A. Manos-Turvey, E.M. Bulloch, P.J. Rutledge, E.N. Baker, J.S. Lott, R.J. Payne, Inhibition studies of Mycobacterium tuberculosis salicylate synthase (Mbtl). ChemMedChem 5, (2010) 1067-1079.

[15] A. Manos-Turvey, K.M. Cergol, N.K. Salam, E.M. Bulloch, G. Chi, A. Pang, W.J. Britton, N.P. West, E.N. Baker, J.S. Lott, R.J. Payne, Synthesis and evaluation of M. tuberculosis salicylate synthase (Mbtl) inhibitors designed to probe plasticity in the active site. Org. Biomol. Chem. 10, (2012) 92239236.

[16] A.J. Harrison, M. Yu, T. Gårdenborg, M. Middleditch, R.J. Ramsay, E.N. Baker, J.S. Lott, The structure of Mbtl from Mycobacterium tuberculosis, the first enzyme in the biosynthesis of the siderophore mycobactin, reveals it to be a salicylate synthase. J. Bacteriol. 188, (2006) 6081-6091.

[17] G. Chi, A. Manos-Turvey, P.D. O'Connor, J.M. Johnston, G.L. Evans, E.N. Baker, R.J. Payne, J.S. Lott, E.M. Bulloch, Implications of binding mode and active site flexibility for inhibitor potency against the salicylate synthase from Mycobacterium tuberculosis. Biochemistry 51, (2012) 4868-4879.

[18] G. Wolber, T. Langer, LigandScout: 3-D pharmacophores derived from protein-bound ligands and their use as virtual screening filters. J. Chem. Inf. Model. 45, (2005) 160-169.

[19] T. Tuccinardi, G. Poli, V. Romboli, A. Giordano, A. Martinelli, Extensive consensus docking evaluation for ligand pose prediction and virtual screening studies. J. Chem. Inf. Model. 54, (2014) 2980-2986.

[20] Poli, G.; Martinelli, A.; Tuccinardi, T. Reliability analysis and optimization of the consensus docking approach for the development of virtual screening studies. J. Enzyme Inhib. Med. Chem. 31, (2016) 167-173.

[21] G. Poli, N. Giuntini, A. Martinelli, T. Tuccinardi, Application of a FLAP-consensus docking mixed strategy for the identification of new fatty acid amide hydrolase inhibitors. J. Chem. Inf. Model. 55, (2015) 667-675.

[22] C. Granchi, A. Capecchi, G. Del Frate, A. Martinelli, M. Macchia, F. Minutolo, T.Tuccinardi, Development and validation of a docking-based virtual screening platform for the identification of new lactate dehydrogenase inhibitors. Molecules 20, (2015) 8772-8790.

[23] T. Tuccinardi, C. Granchi, F. Rizzolio, I. Caligiuri, V. Battistello, G. Toffoli, F. Minutolo, M. Macchia, A. Martinelli, Identification and characterization of a new reversible MAGL inhibitor. Bioorg. Med. Chem. 22, (2014) 3285-3291.

[24] M. Vasan, J. Neres, J. Williams, D.J. Wilson, A.M.Teitelbaum, R.P. Remmel, C.C. Aldrich, Inhibitors of the salicylate synthase (Mbtl) from Mycobacterium tuberculosis discovered by high-throughput screening. ChemMedChem 5, (2010) 2079-2087.

[25] C. Bissantz, B. Kuhn, M. Stahl, A medicinal chemist's guide to molecular interactions. J. Med. Chem. 53, (2010) 5061-5084.

[26] R. Brosch, W.J. Philipp, E. Stavropoulos, M.J. Colston, S.T. Cole, S.V. Gordon, Genomic analysis reveals variation between Mycobacterium tuberculosis H37Rv and the attenuated M. tuberculosis H37Ra strain. Infect. Immun. 67, (1999) 5678-5774.

[27] M. Sritharan, Iron homeostasis in Mycobacterium tuberculosis: mechanistic insight into siderophoremediated iron uptake. J. Bacteriol. 198, (2016) 2399-2409.

[28] B. Schwyn, J.B. Neilands, Universal chemical assay for the detection and determination of siderophores. Anal. Biochem. 160, (1987) 47-56.

[29] F. Porta, A. Gelain, D. Barlocco, N. Ferri, S. Marchianò, V. Cappello, L. Basile, S. Guccione, F. Meneghetti, S. Villa, A field-based disparity analysis of new 1,2,5-oxadiazole derivatives endowed with antiproliferative activity. Chem. Biol. Drug Des. 90, (2017) 820-839.

[30] Korb, O.; Monecke, P.; Hessler, G.; Stützle, T.; Exner, T. E. pharmACOphore: multiple flexible ligand alignment based on ant colony optimization. J. Chem. Inf. Model. 2010, 50, 1669-1681.

[31] O. Korb, T. Stützle, T.E. Exner, Empirical scoring functions for advanced protein-ligand docking with PLANTS. J. Chem. Inf. Model. 49, (2009) 84-96.

[32] D. A. Case, J.T. Berryman, R.M. Betz, D.S. Cerutti, T.E. Cheatham III, T.A. Darden, R.E. Duke, T.J. Giese, H. Gohlke, A.W. Goetz, N. Homeyer, S. Izadi, P. Janowski, J. Kaus, A. Kovalenko, T.S. Lee, S. LeGrand, P. Li, T. Luchko, R. Luo, B. Madej, K.M. Merz, G. Monard, P. Needham, H. Nguyen, H.T. Nguyen, I. Omelyan, A. Onufriev, D.R. Roe, A. Roitberg, R. Salomon-Ferrer, C.L. Simmerling, W. Smith, J. Swails, R.C. Walker, J. Wang, R.M. Wolf, X. Wu, D.M.York, P.A. Kollman, AMBER, version 14. In University of California: San Francisco, CA, 2015.

[33] O. Kerbarh, D.Y. Chirgadze, T.L. Blundell, C. Abell, Crystal structures of Yersinia enterocolitica salicylate synthase and its complex with the reaction products salicylate and pyruvate. J. Mol. Biol. 357, (2006) 524-534.

[34] L. Yu, L. Xu, M. Xu, B. Wan, L. Yu, Q. Huang, Role of $\mathrm{Mg}^{2+}$ ions in protein kinase phosphorylation: insights from molecular dynamics simulations of ATP-kinase complexes, Mol. Simul. 37 (2011) 11431150.

[35] L. Casalino, A. Magistrato, Structural, dynamical and catalytic interplay between $\mathrm{Mg}^{2+}$ ions and RNA. Vices and virtues of atomistic simulations, Inorganica Chim. Acta. 452 (2016) 73-81. 
[36] L. Casalino, G. Palermo, N. Abdurakhmonova, U. Rothlisberger, A. Magistrato, Development of sitespecific Mg2+-RNA force field parameters: A dream or reality? Guidelines from combined molecular dynamics and quantum mechanics simulations, J. Chem. Theory Comput. 13 (2017) 340-352.

[37] H.S. Hayatshahi, D.R. Roe, R. Galindo-Murillo, K.B. Hall, T.E. CheathamllI, Computational assessment of potassium and magnesium ion binding to a buried pocket in GT pase-associating center RNA, J. Phys. Chem. B. 121 (2017) 451-462.

[38] P. Li, B.P. Roberts, D.K. Chakravorty, K.M. Merz, Rational design of particle mesh ewald compatible lennard-jones parameters for +2 metal cations in explicit solvent, J. Chem. Theory Comput. 9 (2013) 2733-2748.

[39] D.M. York, T.A. Darden, L.G. Pedersen, The effect of long-range electrostatic interactions in simulations of macromolecular crystals: a comparison of the Ewald and truncated list methods. J. Chem. Phys. 99, (1993) 8345-8348.

[40] C. Grisostomi, P. Kast, R. Pulido, J. Huynh, D. Hilvert, Efficient in vivo synthesis and rapid purification of chorismic acid using an engineered Escherichia coli strain. Bioorg. Chem. 25, (1997) 297-305.

[41] S. Jasial, Y. Hu, J. Bajorath, How frequently are pan-assay interference compounds active? Largescale analysis of screening data reveals diverse activity profiles, low global hit frequency, and many consistently inactive compounds. J. Med. Chem. 60, (2017) 3879-3886.

[42] J.B. Baell, G.A. Holloway, New substructure filters for removal of pan assay interference compounds (PAINS) from screening libraries and for their exclusion in bioassays. J. Med. Chem. 53, (2010), 27192740.

[43] B.K. Shoichet, Screening in a spirit haunted world. Drug Discov. Today 11, (2006) 607-615.

[44] J.L. Dahlin, J.W. Nissink, J.M. Strasser, S. Francis, L. Higgins, H. Zhou, Z. Zhang, M.A. Walters, PAINS in the assay: chemical mechanisms of assay interference and promiscuous enzymatic inhibition observed during a sulfhydryl-scavenging HTS. J. Med. Chem. 58, (2015) 2091-2113.

[45] M. S. Siegrist, M. Unnikrishnan, M.J. McConnell, M. Borowsky, T. Cheng, N. Siddiqi, S.M. Fortune, D.B. Moody, E.J. Rubin, Mycobacterial Esx-3 is required for mycobactin-mediated iron acquisition. Proc. Natl. Acad. Sci. U. S. A. 106, (2009) 18792-18797.

[46] P.V. Reddy, R.V. Puri, P. Chauhan, R. Kar, A. Rohilla, A. Khera, A.K. Tyagi, Disruption of mycobactin biosynthesis leads to attenuation of Mycobacterium tuberculosis for growth and virulence. J. Infect. Dis. 208, (2013) 1255-1265. 